Supporting Information

\title{
Graphene-induced in-situ growth of monolayer and bilayer 2D SiC crystals toward high-temperature electronics
}

Dechao Geng, ${ }^{1}$ Junping $H u,{ }^{l}$ Wei Fu, ${ }^{2}$ Lay Kee Ang, ${ }^{1}$ Hui Ying Yang ${ }^{l,}$ *

${ }^{1}$ Pillar of Engineering Product Development, Singapore University of Technology and Design, 8

Somapah Road, 487372, Singapore

${ }^{2}$ Department of Chemistry and Centre for Advanced 2D Materials, National University of

Singapore, 3 Science Drive 3, 17543, Singapore

Email: yanghuiying@sutd.edu.sg 

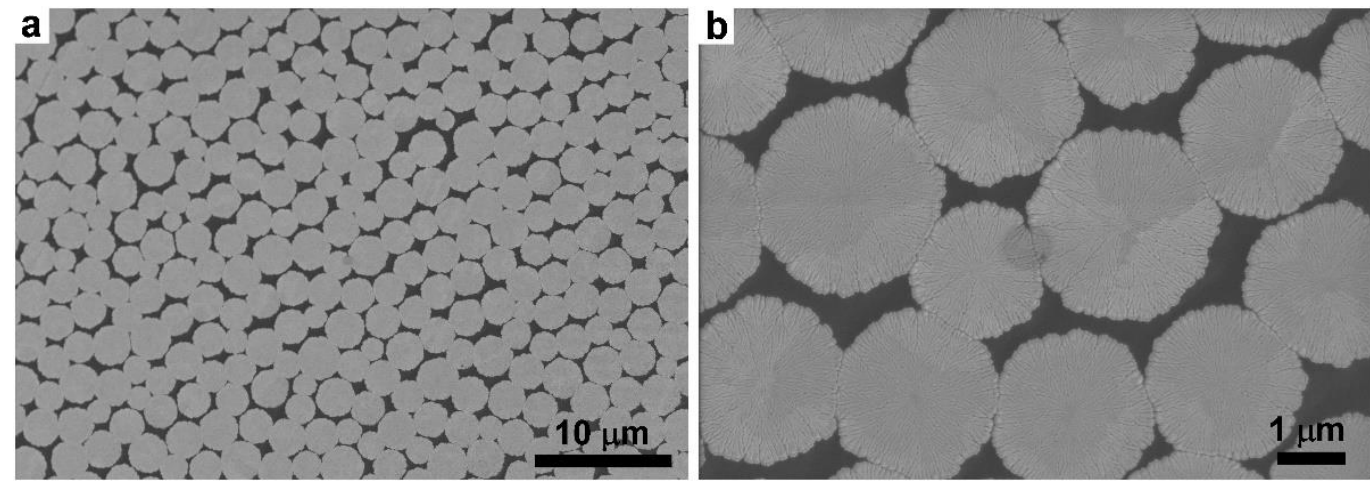

Figure S1. (a) SEM image for large-area deposition of silicon oxides particles on liquid $\mathrm{Cu}$ surface. (b) high resolution SEM image of a typical liquid $\mathrm{Cu}$ surface. Noted that long time annealing can result in formation of silicon oxides particles, while the density can be further modulated.

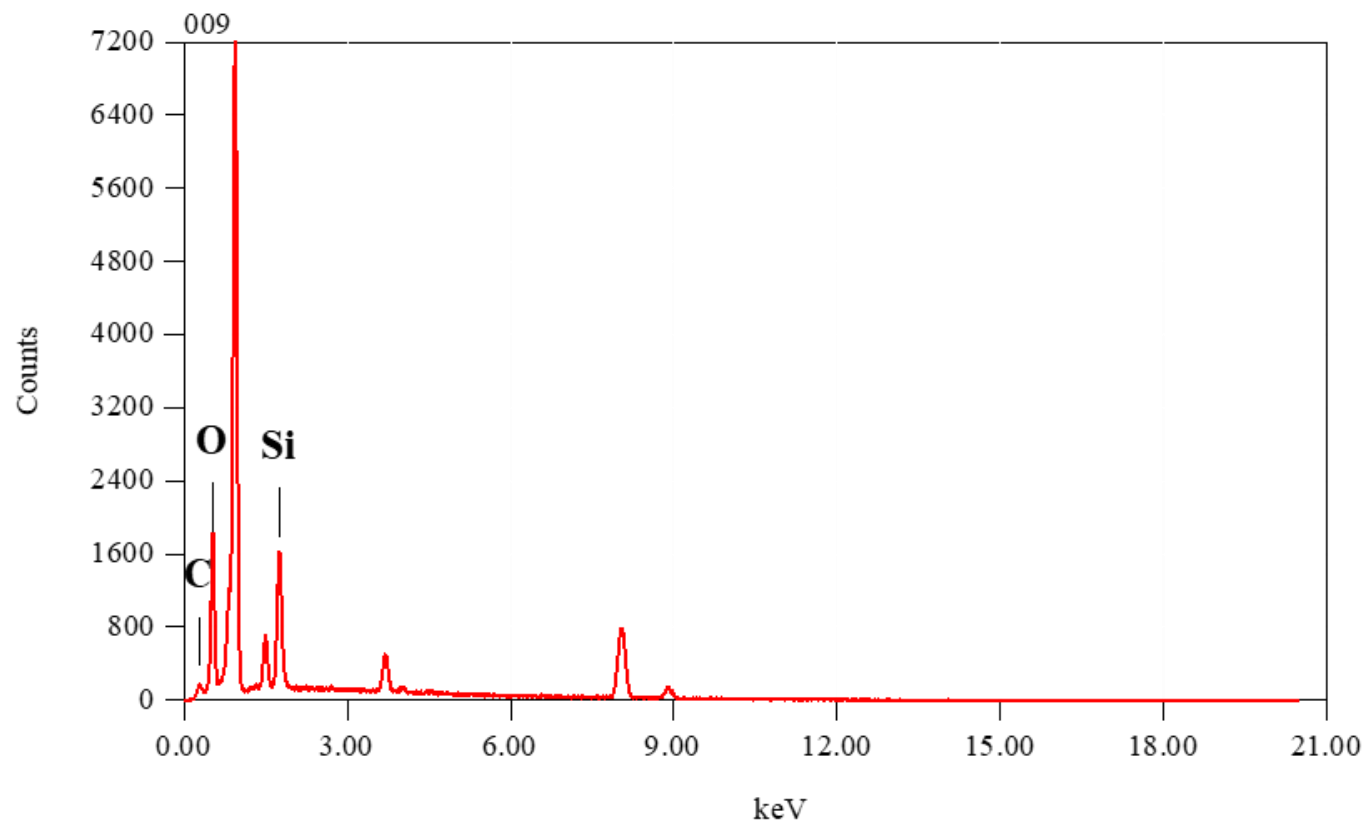

Figure S2. EDX measurements of deposited particles. Both the Si signal and O signal with relatively high intensity are observed, suggesting existence of $\mathrm{Si}$ and $\mathrm{O}$ elements, respectively. The formation of silicon oxides is confirmed based on the EDX results. 

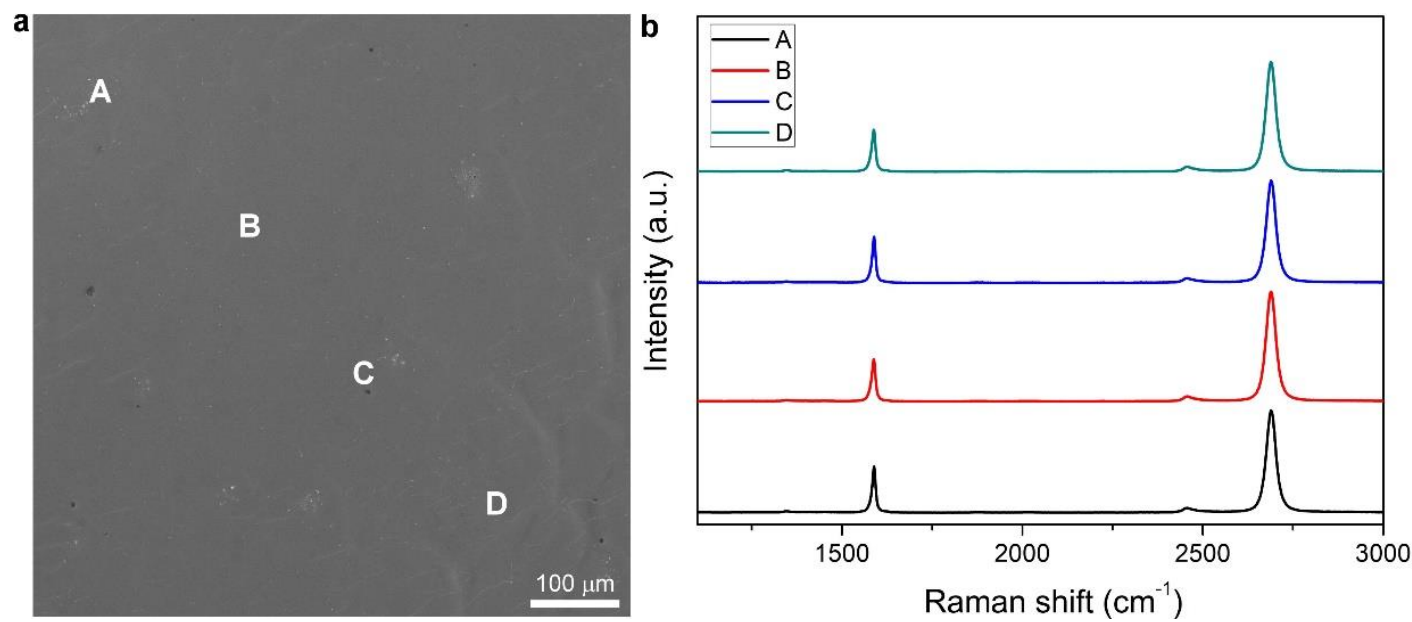

Figure S3. The full coverage of graphene on liquid $\mathrm{Cu}$ surface. (a) SEM image of large-area graphene film onto the surface. (b) Raman spectrum of the labelled dots in (a), indicating the full coverage of monolayer graphene film.
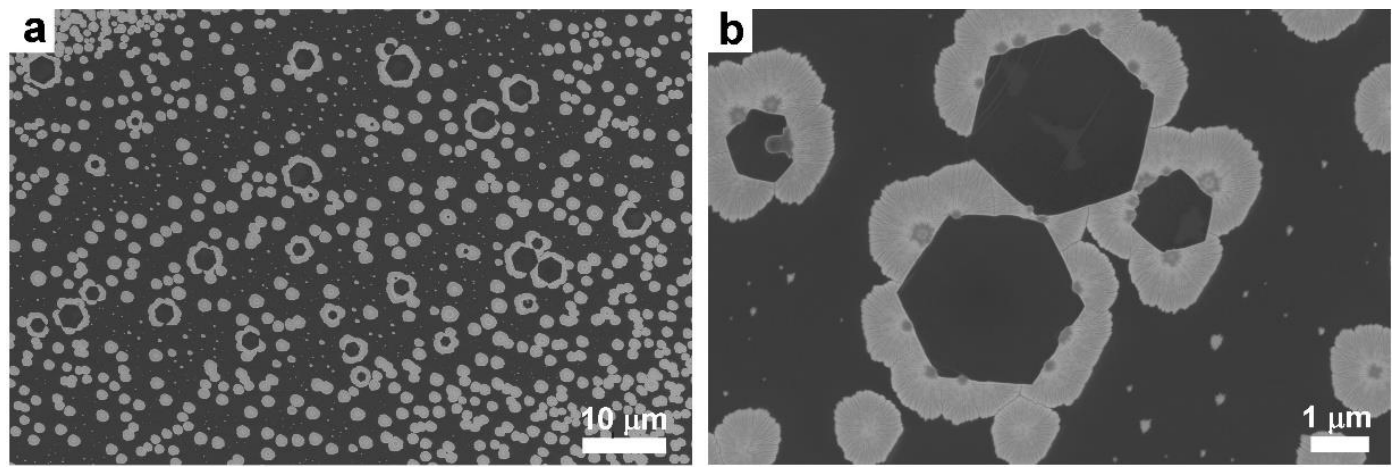

Figure S4. (a) SEM image for graphene growth on the as-formed silicon oxides on liquid $\mathrm{Cu}$ surface. (b) high resolution SEM image of typical graphene flakes. Noted that graphene are almost nucleated and grown on the silicon oxides particles. 

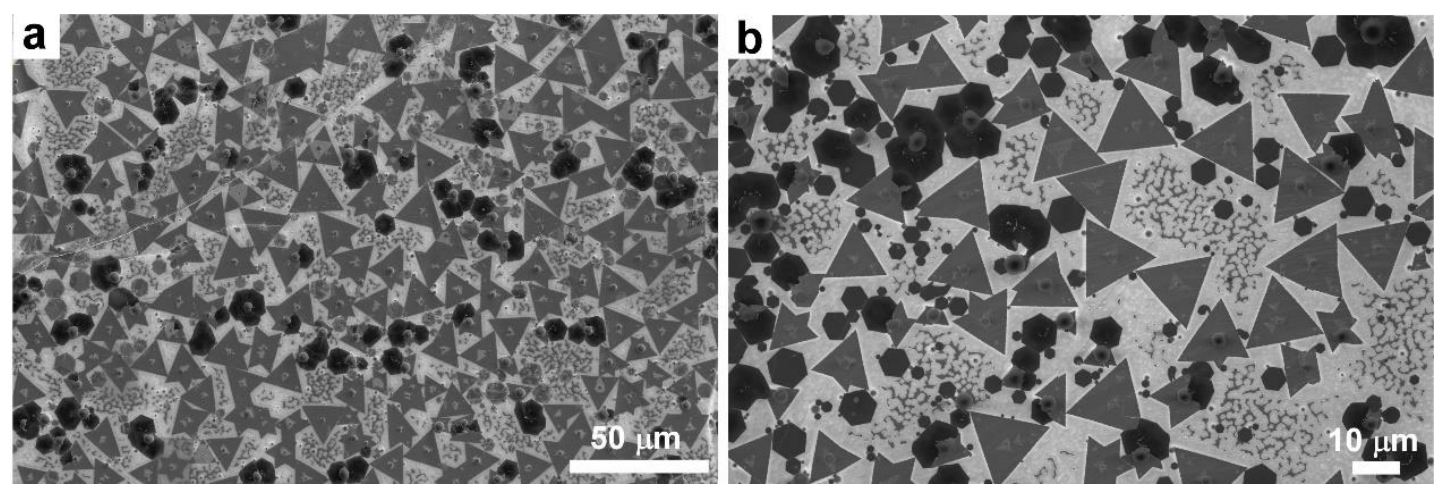

Figure S5. SEM images of triangular SiC crystals (a and b). It is seen that the SiC crystals are uniformly dispersed onto the whole surface, indicating uniform growth.

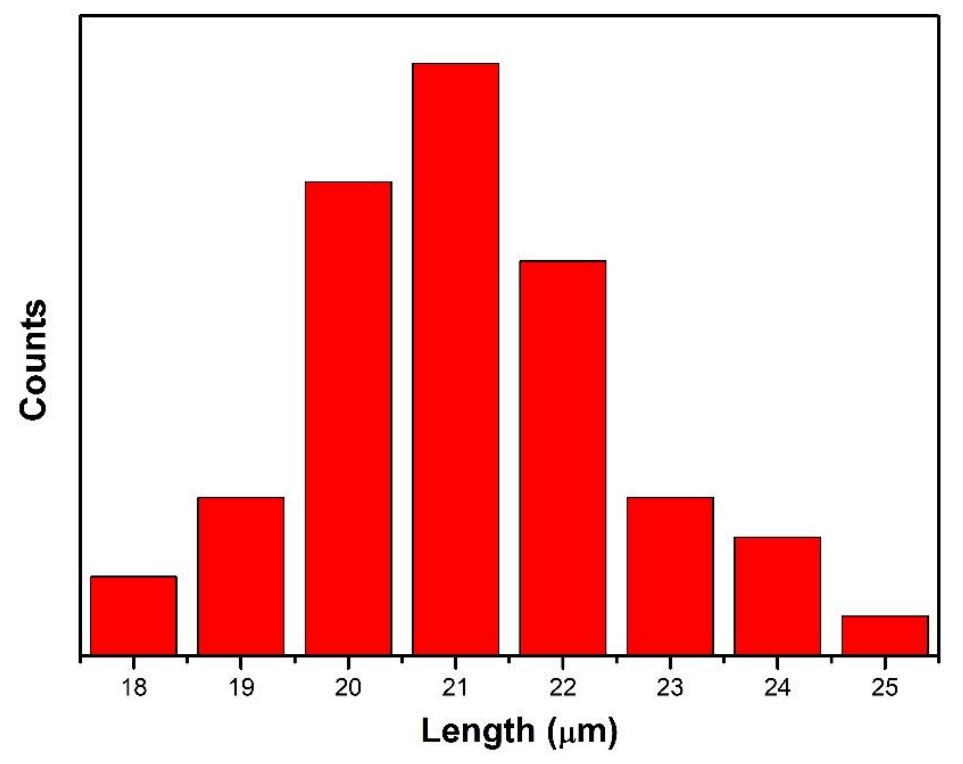

Figure S6. Size statistics of the as-formed SiC crystals. Noted that the size is uniform with a narrow range, almost about $21 \mu \mathrm{m}$. 

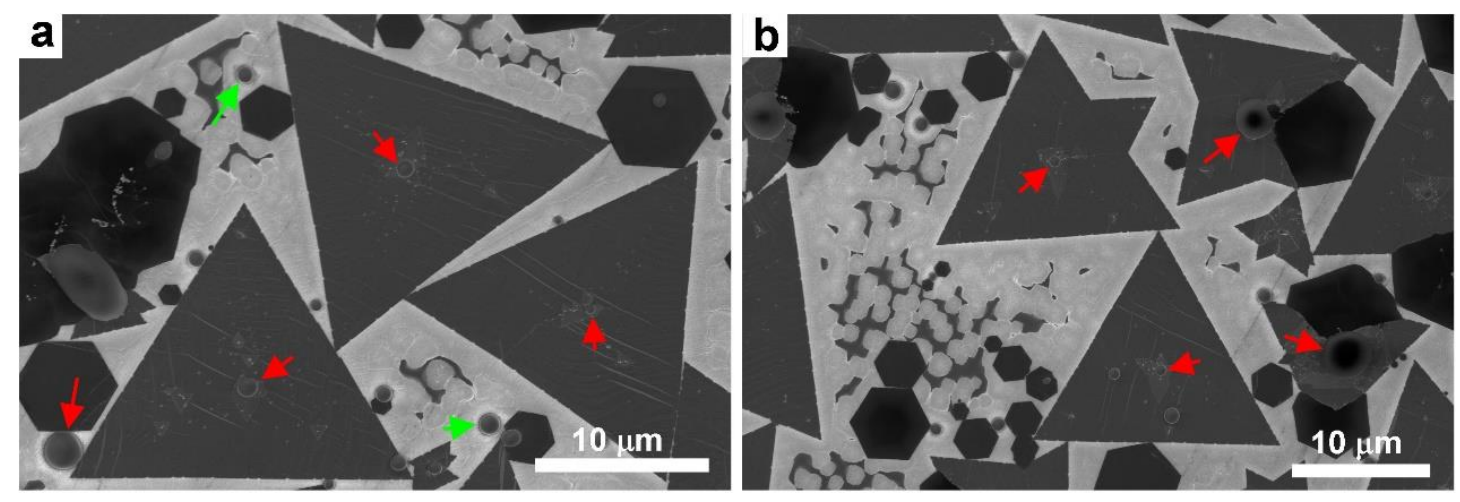

Figure S7. SEM images of the located silicon oxides particles. (a) The as-formed triangular SiC crystals, on which silicon oxides as red arrow marked are located at center areas. (b) The middle-staged SiC crystals, on which larger sized silicon oxides particles are directly observed and then gradually became smaller once fully into SiC crystals.
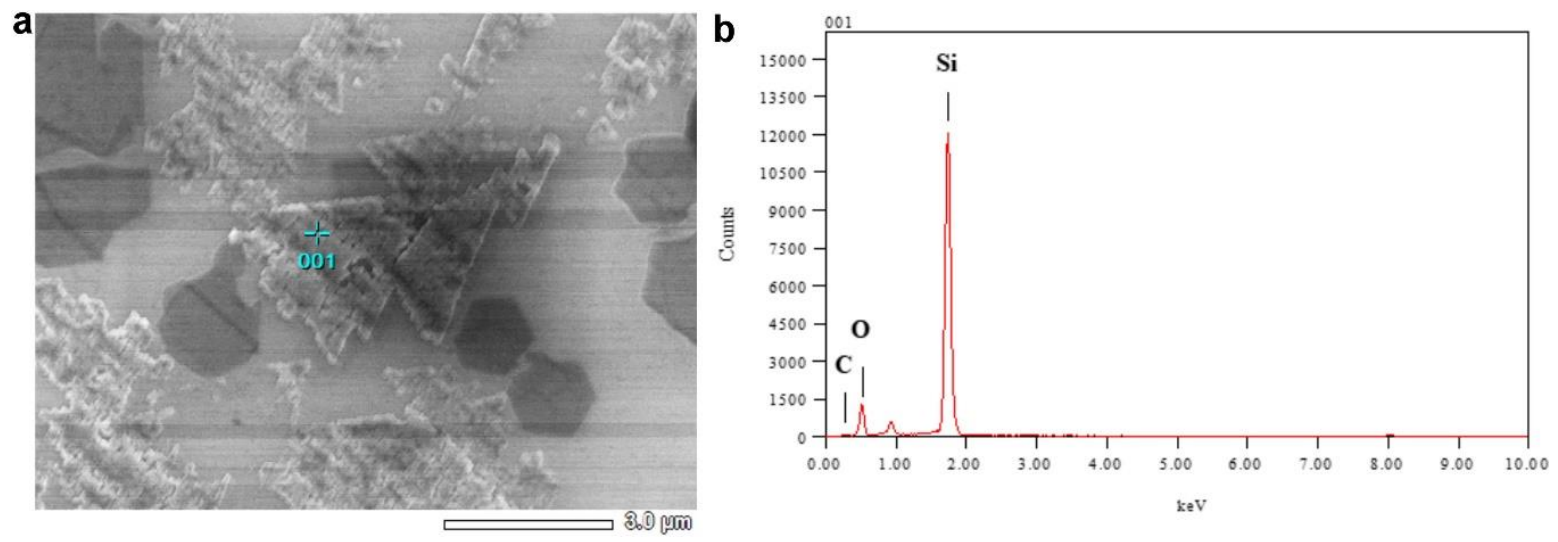

Figure S8. Elements analysis of the as-transferred SiC crystals. (a) SEM image of the as-transferred triangular SiC flakes. (b) EDX measurement of the located point in (a), showing the presence of silicon, carbon and oxygen. 

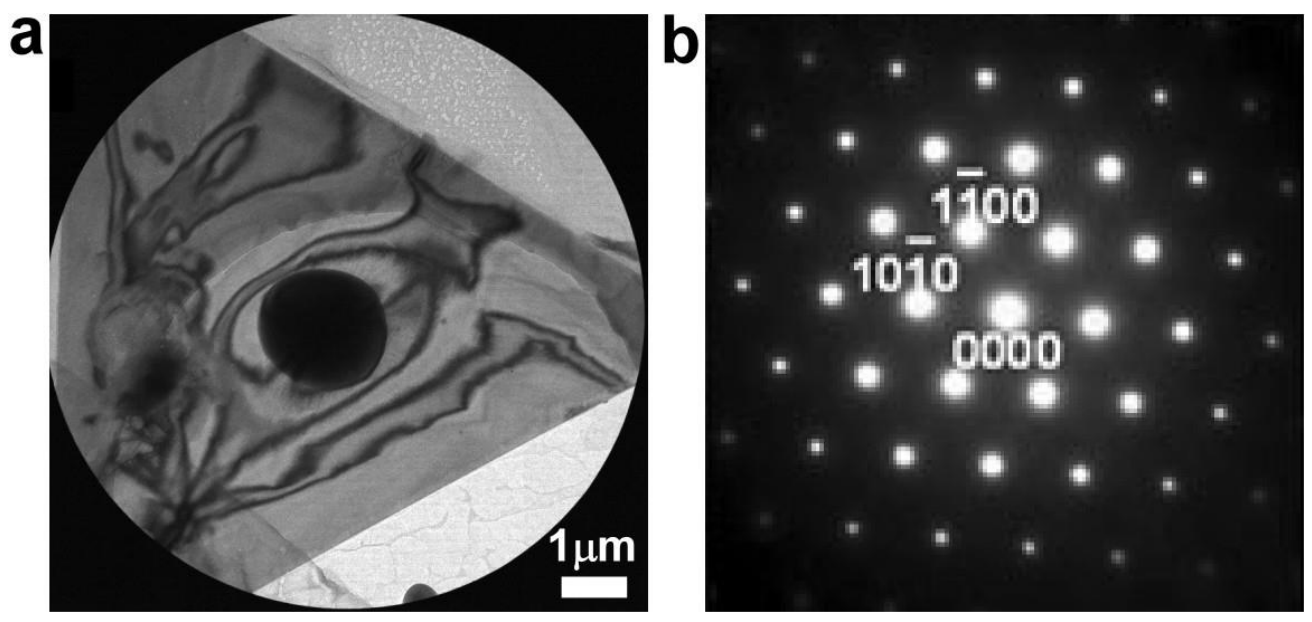

Figure S9. TEM characterization of the $\mathrm{SiC}$ flake. (a) TEM image of triangular $\mathrm{SiC}$ crystals. (b) SAED patterns of the SiC flakes, indicating a high crystallinity.
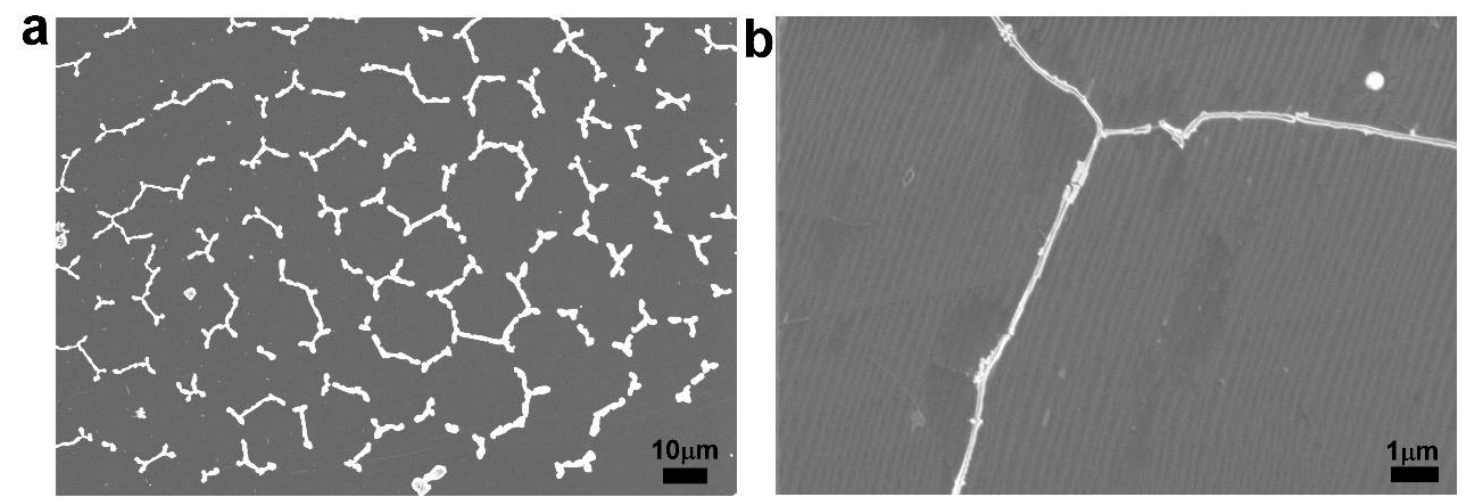

Figure S10. The control experiment on solid $\mathrm{Cu}$ surface. (a and b) The as-grown graphene and silicon oxides on solid $\mathrm{Cu}$ surface, whereas no $\mathrm{SiC}$ crystals were detected, further indicating the unique role of liquid $\mathrm{Cu}$ surface in shaping $\mathrm{SiC}$. 

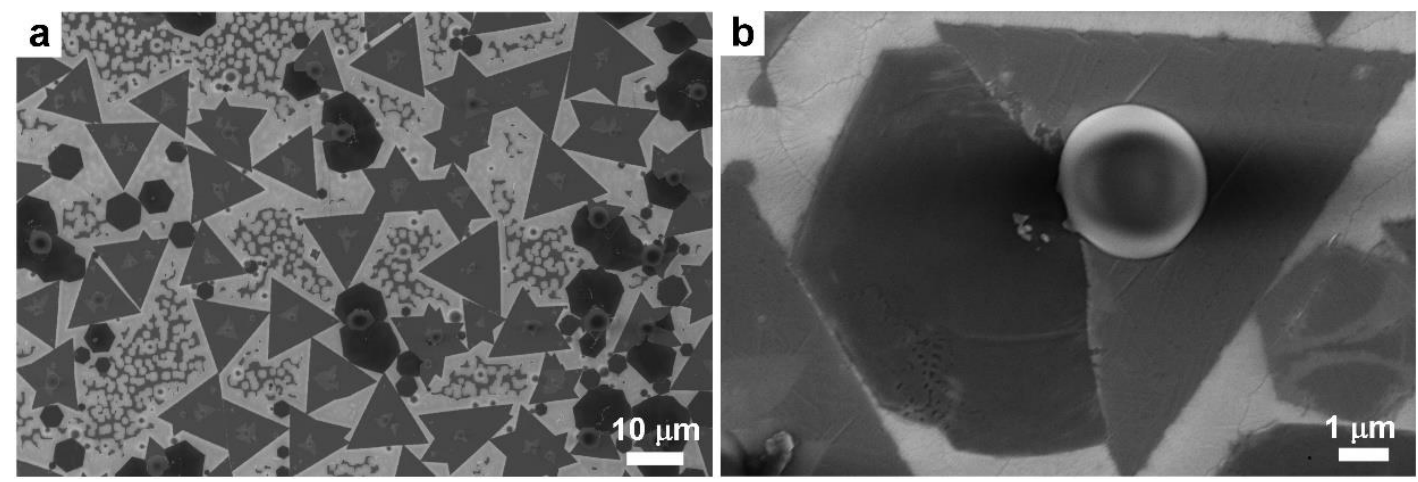

Figure S11. ( $a$ and b) SEM images of the middle staged SiC crystals.
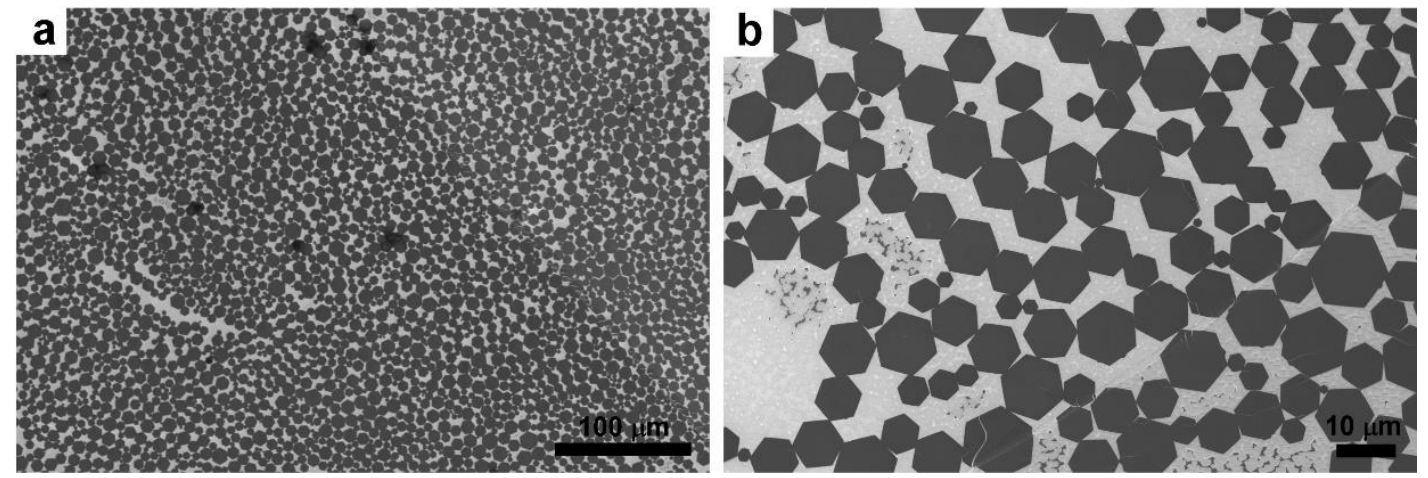

Figure S12. (a and b) Control experiments of the in-situ growth. SEM images of the hexagonal graphene on liquid $\mathrm{Cu}$ surface. 

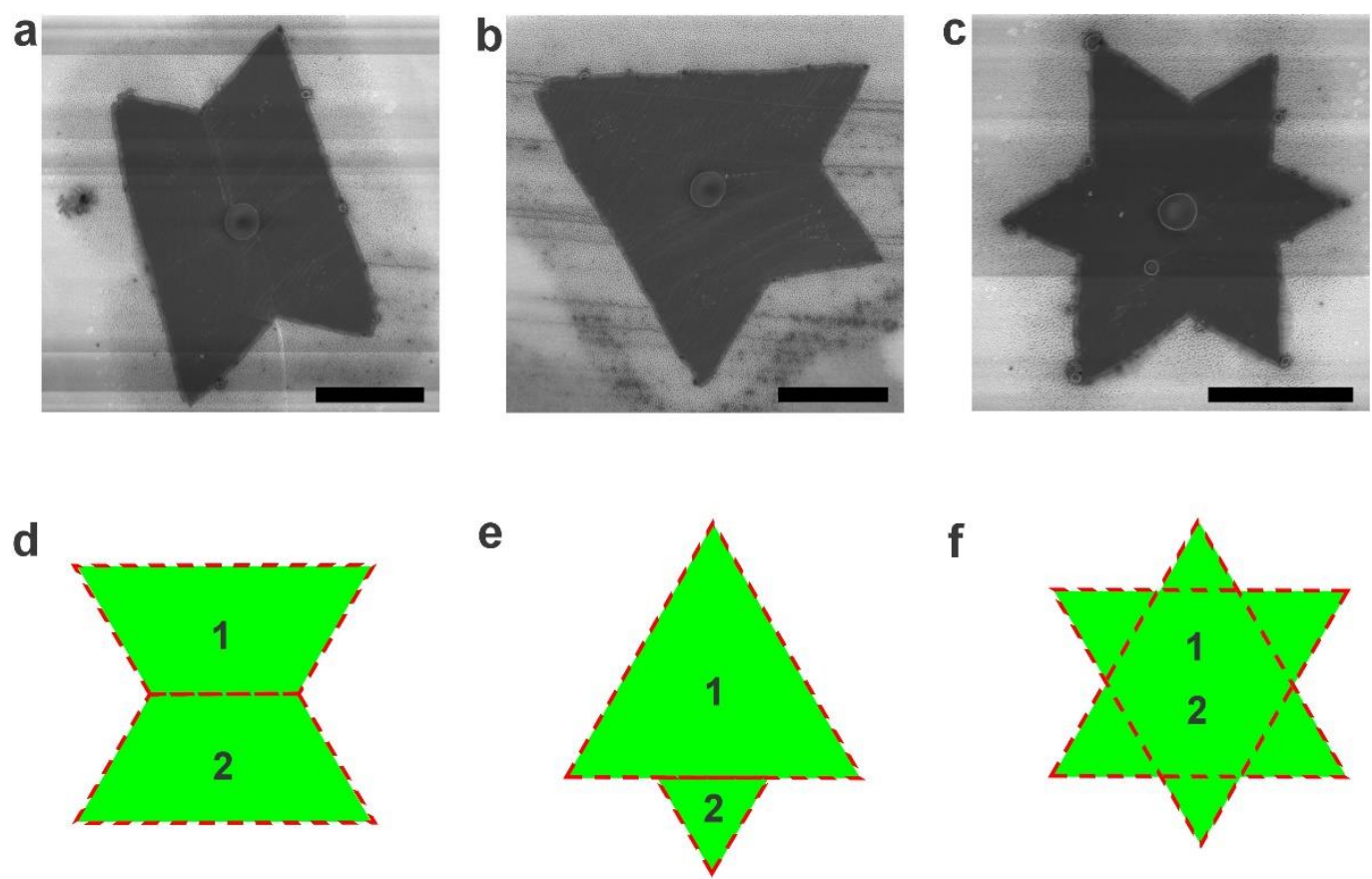

Figure S13. Morphology-engineering of SiC crystals. (a-c) The SEM images of pointed SiC flakes. (d-f) The formation scheme of the corresponding shaped SiC, whereas nuclei are labelled. It is noted that all the shaped $\mathrm{SiC}$ crystals are composed of triangular building blocks. All the scale bars are $5 \mu \mathrm{m}$.
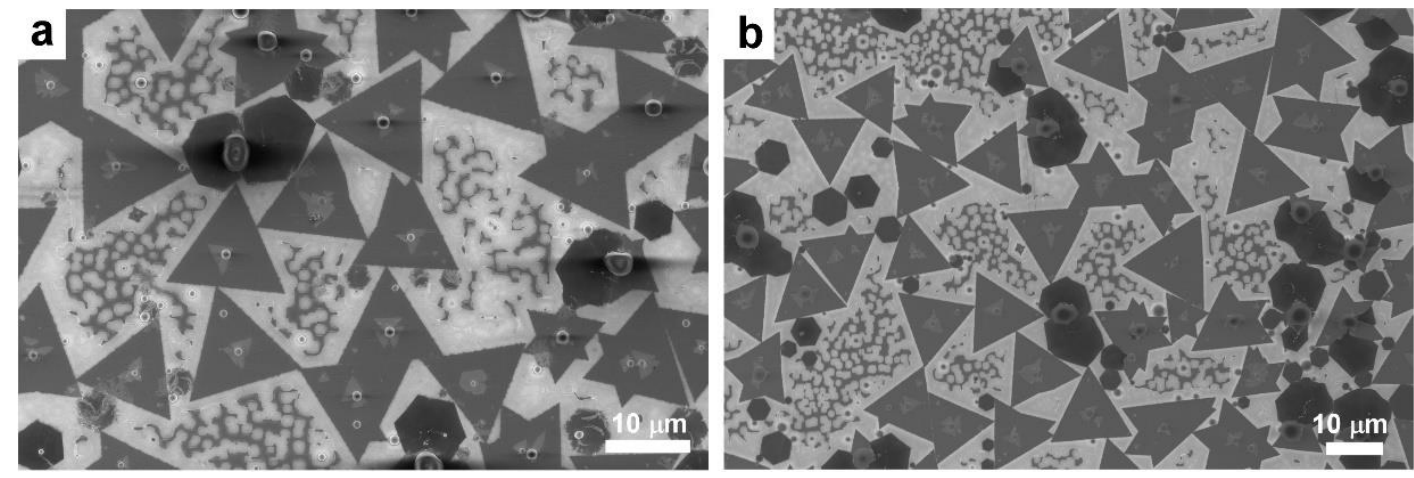

Figure S14. The growth of bilayer SiC crystals. (a and b) SEM images of the as-grown bilayer $\mathrm{SiC}$ crystals. Noted that nearly all the triangular $\mathrm{SiC}$ crystals have the second growth, leading to formation of bilayer $\mathrm{SiC}$. 

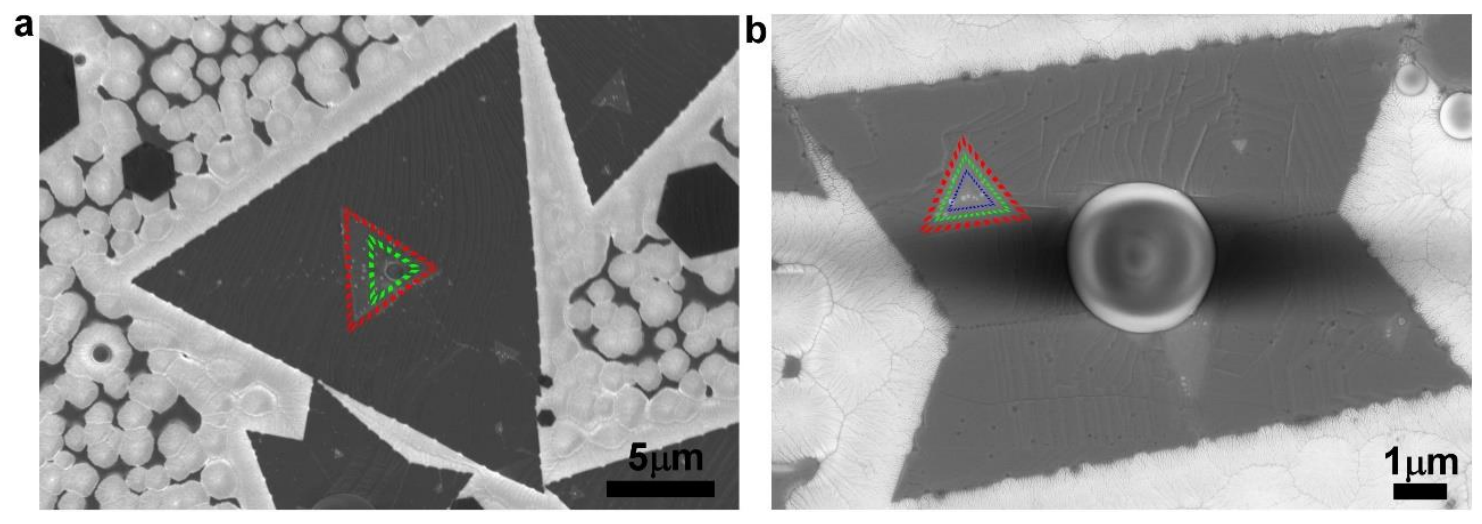

Figure S15. Growth of the few-layer SiC crystals. (a) Tri-layer SiC and (b) Four-layer SiC crystals are observed on liquid $\mathrm{Cu}$ surface.

\section{Computational details:}

All calculations are based on density functional theory (DFT) using the plane-wave pseudopotentials $^{[\mathrm{i}-\mathrm{ii}]}$ associated with the exchange-correlation formed by Perdew-BurkeErnzerhof $(\mathrm{PBE})^{[\mathrm{iii}-\mathrm{iv}]}$ as implemented in the Vienna Ab initio Simulation Package (VASP). ${ }^{\text {[v] }}$ The cutoff energy is set to $500 \mathrm{eV}$ for plane wave expansion of the wave functions. For $\mathrm{SiO}_{2}$, the Brillouin zone is sampled using a $7 \times 7 \times 7$ Monkhorst-Pack $k$-point grid $^{[\text {vi] }}$ for the structural optimization. For graphene, $\mathrm{SiC}$, and $\mathrm{CO}_{2}$, the Monkhorst-Pack $k$-point grid of $7 \times 11 \times 3,9 \times 9$ $\times 9,3 \times 3 \times 3$ are utilized to sample the Brillouin zone respectively. The convergence criteria for total energy and ionic forces are set to $10^{-6} \mathrm{eV}$ and $0.0001 \mathrm{eV} / \AA$, respectively. The structural pattern with a $\sim 15 \AA$ vacuum region in all directions to minimize the interaction between adjacent images for $\mathrm{CO}_{2}$ gas molecular and with a $\sim 15 \AA$ vacuum region in the $\mathrm{z}$ directions to minimize the interaction between adjacent images for the graphene. 


\section{The reaction activation energy:}

We here assume the following reaction:

$$
\mathrm{SiO}_{2}+\mathrm{C} \leftrightarrow \mathrm{SiC}+\mathrm{CO}_{2}
$$

The difference in total energies between reactants and products is used to determine the reaction activation energy. The volume and entropy effects are usually negligible during the reaction. Therefore, the reaction activation energy can be approximately from the energy difference:

$$
E_{\text {reac }}=E_{S i C}+E_{\mathrm{CO}_{2}}-E_{\mathrm{SiO}_{2}}-E_{C}
$$

where $E_{\mathrm{SiC}}, E_{\mathrm{CO}_{2}}, E_{\mathrm{SiO}_{2}}$, and $E_{\mathrm{C}}$ are the total energies of the reactants and products, and the $E_{\text {reac }}$ represents the activation energy of this reaction. The negative value of $E_{\text {reac }}$ stands for the reaction can occur spontaneously, but not otherwise. After calculations, when the reaction contains only one $\mathrm{Si}$ atom, two $\mathrm{O}$ atoms and one $\mathrm{C}$ atom, the activation energy of the reaction is $-5.09 \mathrm{eV}$, indicating that this reaction can occur spontaneously. The following is the structural information.

\section{Structural information for $\mathrm{SiO}_{2}$, graphene, $\mathrm{SiC}$ and $\mathrm{CO}_{2}$ :}

The detailed atomic information is following:

1. CONTCAR-SiO ${ }_{2}$ :

$\mathrm{SiO}_{2}$

1.00000000000000 


$\begin{array}{ccc}7.4587406825739588 & 0.00000000000000000 & 0.0000000000000000 \\ -0.00000000000000000 & 7.4587406825739588 & -0.0000000000000000 \\ & & \\ 0.0000000000000000 & -0.0000000000000000 & 7.4587406825739588\end{array}$

$\mathrm{Si} \quad \mathrm{O}$

$8 \quad 16$

Direct

$-0.0000000000000000-0.00000000000000000-0.0000000000000000$

$\begin{array}{llll}-0.0000000000000000 & 0.5000000000000000 & 0.5000000000000000\end{array}$

$0.5000000000000000 \quad 0.5000000000000000-0.0000000000000000$

$\begin{array}{llll}0.5000000000000000 & 0.0000000000000000 & 0.5000000000000000\end{array}$

$0.7500000000000000 \quad 0.2500000000000000 \quad 0.7500000000000000$

$\begin{array}{llll}0.2500000000000000 & 0.2500000000000000 & 0.2500000000000000\end{array}$

$0.2500000000000000 \quad 0.7500000000000000 \quad 0.7500000000000000$

$\begin{array}{llll}0.7500000000000000 & 0.7500000000000000 & 0.2500000000000000\end{array}$

$\begin{array}{llll}0.1250000000000000 & 0.1250000000000000 & 0.1250000000000000\end{array}$

$\begin{array}{llll}0.8750000000000000 & 0.3750000000000000 & 0.6250000000000000\end{array}$

$0.3750000000000000 \quad 0.6250000000000000 \quad 0.8750000000000000$ 

0.6250000000000000
0.8750000000000000
0.3750000000000000
0.3750000000000000
0.8750000000000000
0.6250000000000000
0.8750000000000000
0.6250000000000000
0.3750000000000000
0.6250000000000000
0.3750000000000000
0.8750000000000000
0.1250000000000000
0.6250000000000000
0.6250000000000000
0.8750000000000000
0.8750000000000000
0.1250000000000000
0.3750000000000000
0.1250000000000000
0.3750000000000000
0.3750000000000000
0.3750000000000000
0.1250000000000000
0.8750000000000000
0.1250000000000000
0.8750000000000000
0.6250000000000000
0.1250000000000000
0.6250000000000000
0.1250000000000000
0.8750000000000000
0.8750000000000000
0.1250000000000000
0.3750000000000000
0.3750000000000000
0.6250000000000000
0.6250000000000000
0.1250000000000000

2. CONTCAR-graphene:

graphene

1.0

4.9200000763

0.0000000000

0.0000000000 
$-1.2300000191$

0.0000000000

C

4

Direct
0.000000000
0.000000000
0.500000000
0.166669995
0.666670024
0.500000000
0.500000000
0.000000000
0.500000000
0.666670024
0.666670024
0.500000000

\section{CONTCAR-SiC:}

$\mathrm{SiC}$

1.00000000000000

$\begin{array}{lll}4.3772223968613009 & 0.0000000000000000 & -0.0000000000000000\end{array}$

$\begin{array}{lll}0.0000000000000000 & 4.3772223968613009 & 0.0000000000000000\end{array}$

$0.0000000000000000 \quad-0.0000000000000000 \quad 4.3772223968613009$

C Si

$4 \quad 4$

S-13 
Direct

$$
\begin{array}{llll}
0.2500000000000000 & 0.2500000000000000 & 0.2500000000000000 \\
0.7500000000000000 & 0.7500000000000000 & 0.2500000000000000 \\
0.7500000000000000 & 0.2500000000000000 & 0.7500000000000000 \\
0.2500000000000000 & 0.7500000000000000 & 0.7500000000000000 \\
0.0000000000000000 & 0.0000000000000000 & 0.0000000000000000 \\
0.0000000000000000 & 0.5000000000000000 & 0.5000000000000000 \\
0.5000000000000000 & 0.0000000000000000 & 0.5000000000000000 \\
0.5000000000000000 & 0.5000000000000000 & 0.0000000000000000
\end{array}
$$

4. CONTCAR-CO ${ }_{2}$ :

$\mathrm{CO} 2$

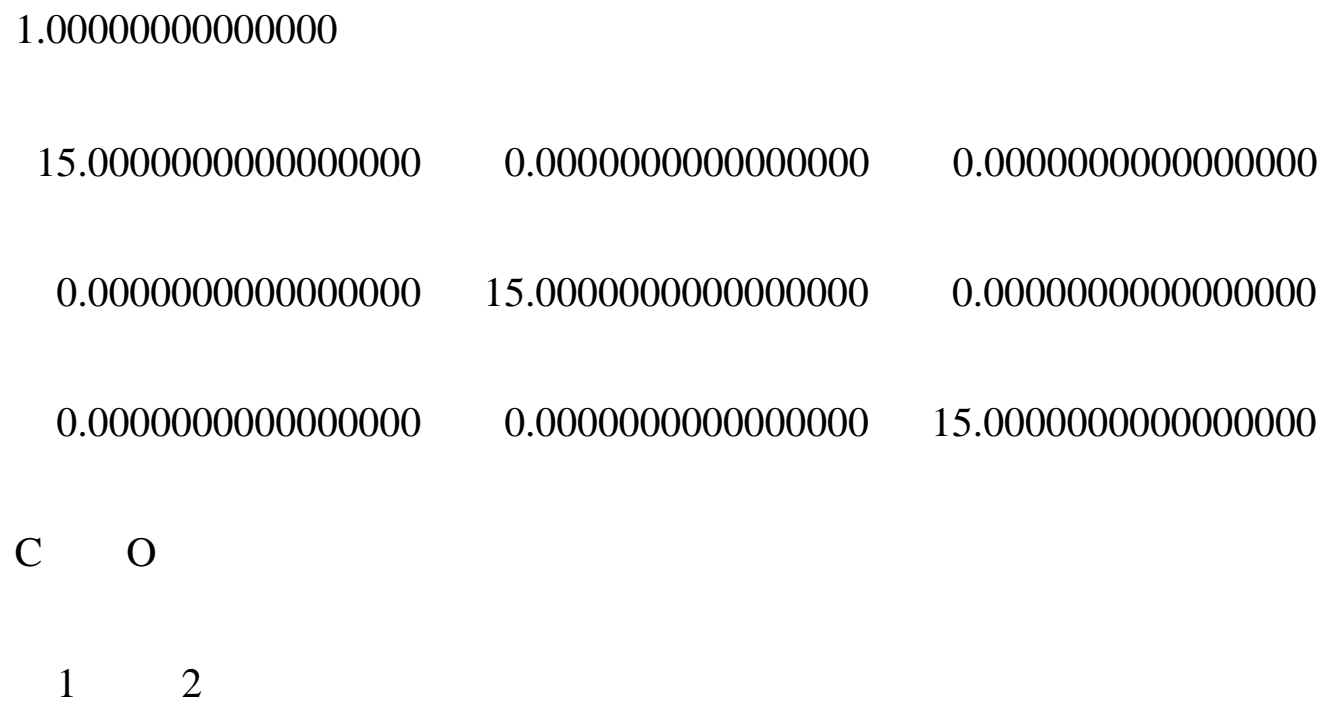


Direct

$$
\begin{array}{llll}
0.4999977782338629 & 0.5000000000000000 & 0.5000000000000000 \\
0.5784221104155960 & 0.5000000000000000 & 0.5000000000000000 \\
0.4215701273505532 & 0.5000000000000000 & 0.5000000000000000
\end{array}
$$

\section{Reference}

[1] G. Kresse, J. Joubert, Phys. Rev. B. 1999 59, 1758.

[2] P. E. Blöchl, Phys. Rev. B. 1994, 50, 17953.

[3] G. Kresse, J. Hafner, Phys. Rev. B. 1993, 47, 558.

[4] G. Kresse, J. Furthmuller, Phys. Rev. B. 1996, 54, 11169.

[5] J. P. Perdew, K. Burke, M. Ernzerhof, Phys. Rev. Lett. 1996, 77, 3865.

[6] H. J. Monkhorst, J. D. Pack, Phys. Rev. B. 1976, 13, 5188. 